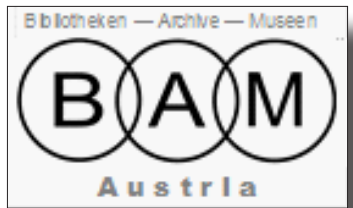

\title{
BAM AUSTRIA: ARBEITSKREIS BIBLIOTHEKEN, ARCHIVE, MU- SEEN ÖSTERREICHS: AKTIVITÄTEN 2016
}

von Bruno Bauer, Markus Feigl, Gabriele Fröschl, Martina Griesser, Rainer Hubert, Lorenz Mikoletzky, Alfred Schmidt, Siegfried Steinlechner, Harald Wendelin

Im Jahr 2016 fanden im Marchettischlössl der Österreichischen Mediathek drei Sitzungen von BAM-Austria statt: am 5. April, 4. Oktober und 28. November.

Im Berichtsjahr hat Gabriele Fröschl, Vertreterin der Medienarchive Österreichs (MAA), die Funktion der BAM-Sprecherin von Bruno Bauer, Vertreter der VÖB, übernommen; als Stellvertreter fungiert Alfred Schmidt, Vertreter der Österreichischen Nationalbibliothek.

Im 2016 erschienenen Tagungsband zum Bibliothekartag Wien 2015 wurden zwei Beiträge der BAM-Session - von Dietrich Schüller und Rainer Hubert ${ }^{1}$ sowie von Harald Weigel ${ }^{2}$ - veröffentlicht.

\section{GND-Umfrage an BAM-Einrichtungen}

Aufbauend auf eine deutsche Initiative, die das Ziel verfolgt, die Gemeinsame Normdatei (GND) auch für Archive und Museen zu öffnen, wurde die GND zum Leitthema von BAM-Austria für 2016 erhoben. ${ }^{3}$ Um einen fundierten Überblick über die Praxis der Nutzung der GND, aber auch weiterer inhaltlicher Erschließungsinstrumente an Bibliotheken, Archiven und Museen in Österreich zu erhalten, wurde eine Projektarbeit im Rahmen des Universitätslehrgangs „Library and Information Studies“ an der Österreichischen Nationalbibliothek angeregt. Unter der Projektleitung von Josef Steiner, Leiter der Hauptabteilung Bestandsaufbau und Bearbeitung der ÖNB, wurde vom Projektteam Olga Kmyta, Irina Sucker und Barbara Markovic ein Fragebogen zu diesem Thema ausgearbeitet. Dieser wurde im Rahmen einer BAM-Sitzung vorgestellt und die Mitglieder von 
BAM übermittelten in der Folge Feedback an die Projektgruppe und unterstützten auch die Erstellung einer Adressenliste von Bibliotheken, Archiven und Museen, denen der Fragebogen zugesandt werden sollte. Von BAM-Austria wurde angeregt, dass der Fragebogen nicht zu sehr auf die GND ausgerichtet werden sollte, sondern der Fokus vielmehr generell auf der Erhebung der gängigen Praktiken der Objekterschließung an Bibliotheken, Archiven und Museen in Österreich sowie der jeweils eingesetzten Regelwerke gelegt werden sollte. Abgefragt wurde auch die Einschätzung der einzelnen Einrichtungen zum Themenkomplex Open Data. Es war diese die erste gemeinsam für den BAM-Bereich durchgeführte Befragung. Von 198 angeschriebenen Institutionen antworteten 62 (31\% Rücklaufquote), davon 20 Bibliotheken, 14 Archive, 7 Museen und 21 kombinierte Einrichtungen. $75 \%$ der an der Befragung teilnehmenden Institutionen verfügen über ein kontrolliertes (normiertes) Vokabular, $35 \%$ verwenden die GND.

Die Ergebnisse der Umfrage wurden von der Projektgruppe als Studie vorgelegt und in einer BAM-Sitzung präsentiert. In komprimierter Form wurde sie auch in den Mitteilungen der VÖB 3/4 (2016) veröffentlicht. ${ }^{4}$

Von BAM-Austria ergeht an dieser Stelle ein herzliches Dankeschön an die Projektgruppe und den Projektleiter für die geleistete Arbeit; die vorgelegte Studie bietet einen fundierten Überblick über die gängige Praxis der Objekterschließung an Bibliotheken, Archiven und Museen und stellt somit eine ausgezeichnete Grundlage für weitere gemeinsame Schritte der Gedächtnisorganisationen im Bereich der Metadaten dar.

\section{Vorbereitungen für BAM-Workshop 2017 zum Thema Metadaten}

Die ursprünglich von BAM-Austria verfolgte Idee, eine gemeinsame Informationsveranstaltung zum Thema GND durchzuführen, wurde aufgrund der Ergebnisse der Befragung wieder verworfen - die GND hat zwar einen sehr hohen Verbreitungsgrad an Bibliotheken, nicht aber an Archiven und Museen. Weil die im Rahmen der Umfrage gestellte Frage „Wären Sie an einer Vernetzungsveranstaltung zu Open Data interessiert?" überwiegend mit ja beantwortet worden war, wurde im Spätherbst 2016 von BAM-Austria mit der Vorbereitung für einen Workshop zum Thema Metadaten und Linked Open Data (LOD) begonnen, der für 11. Mai 2017 geplant ist. Im Workshop sollen der Mehrwert von LOD für die einzelnen Institutionen dargestellt sowie Best Practice-Anwendungen vorgestellt werden. 


\section{Vorschau BAM-Session am Österreichischen Bibliothekartag in Linz 2017}

Aufgrund der positiven Erfahrungen mit der Beteiligung von BAM-Austria am Österreichischen Bibliothekartag in Wien 2015 wird auch im Rahmen des Österreichischen Bibliothekartags in Linz 2017 eine BAM-Session vorbereitet. Ziel ist es, ein Angebot zu einem für alle BAM-Einrichtungen gleichermaßen wichtigen Themen (Metadaten) vorzubereiten, das in verschiedenen Vorträgen aus dem unterschiedlichen Blickwinkel der in BAM vertretenen Institutionen beleuchtet wird.

Mag. Bruno Bauer

ORCID: http://orcid.org/0000-0002-4729-331X Vorstandsmitglied der Vereinigung Österreichischer Bibliothekarinnen und Bibliothekare (VÖB) Universitätsbibliothek der Medizinischen Universität Wien E-Mail: bruno.bauer@meduniwien.ac.at

Mag. Markus Feigl Geschäftsführer des Büchereiverband Österreichs (BVÖ) E-Mail: feigl@bvoe.at

Mag. ${ }^{a}$ Dr. ${ }^{\text {in }}$ Gabriele Fröschl Vertretung der Medienarchive Austria (MAA)

Österreichische Mediathek E-Mail: gabriele.froeschl@mediathek.at

Dr. ${ }^{\text {in }}$ Martina Griesser

Technisches Museum

E-Mail: martina.griesser@tmw.at

Dr. Rainer Hubert Vertretung der Medienarchive Austria (MAA) Österreichische Mediathek E-Mail: rainer.hubert@chello.at

Hon.Prof. Dr. Lorenz Mikoletzky Verband Österreichischer Archivarinnen und Archivare (VÖA)

Österreichisches Staatsarchiv E-Mail: lorenz.mikoletzky@oesta.gv.at 
Dr. Alfred Schmidt

Österreichische Nationalbibliothek E-Mail: alfred.schmidt@onb.ac.at

Mag. Siegfried Steinlechner ORF-TV / Kultur E-Mail: siegfried.steinlechner@orf.at

Dr. Harald Wendelin Technisches Museum Wien E-Mail: harald.wendelin@tmw.at

1 Schüller, Dietrich; Hubert, Rainer: Memory of the World: Das UNESCOProgramm zur Sicherung des Dokumentenerbes. In: Bauer, Bruno; Ferus, Andreas; Pauser, Josef (Hrsg.): Offen(siv)e Bibliotheken. Neue Zugänge, neue Strukturen, neue Chancen: 32. Österreichischer Bibliothekartag. Wien, 15.-18. September 2015. Graz; Feldkirch: Wolfgang Neugebauer Verlag GmbH, 2016. (Schriften der Vereinigung Österreichischer Bibliothekarinnen und Bibliothekare (VÖB); 14), S. 59-65.

2 Weigel, Harald: BAM Austria - Einheit in Vielfalt? Kulturbewahrer und Informationsvermittler: einzeln stark - gemeinsam stärker. In: Bauer, Bruno; Ferus, Andreas; Pauser, Josef (Hrsg.): Offen(siv)e Bibliotheken. Neue Zugänge, neue Strukturen, neue Chancen: 32. Österreichischer Bibliothekartag. Wien, 15.-18. September 2015. Graz; Feldkirch: Wolfgang Neugebauer Verlag GmbH, 2016. (Schriften der Vereinigung Österreichischer Bibliothekarinnen und Bibliothekare (VÖB); 14), S. 67-82.

3 Bauer, Bruno; Fröschl, Gabriele; Hubert, Rainer; Leitner, Gerald; Mikoletzky, Lorenz; Schmidt, Alfred; Steinlechner, Siegried: BAM Austria: Bibliotheken, Archive, Museen Österreichs: Aktivitäten 2015. In: Mitteilungen der Vereinigung Österreichischer Bibliothekarinnen \& Bibliothekare 68 (2015), H. 3/4, S. 621-625. Online unter: https://ojs.univie.ac.at/index.php/voebm/article/view/1302

4 Marković, Barbara; Kmyta, Olga; Sucker, Irina: Objekterschließung an Bibliotheken, Museen und Archiven in Österreich. Ergebnisse einer Erhebung. In: Mitteilungen der Vereinigung Österreichischer Bibliothekarinnen \& Bibliothekare 69 (2016), H. 3/4, S. 414-421. Online unter: https://ojs.univie.ac.at/index.php/voebm/article/view/1731

Dieses Werk ist lizenziert unter einer

Creative-Commons-Lizenz Namensnennung 4.0 International 